\title{
Análise comparativa da região codificadora para a proteína capsidial de isolados de PepYMV e PVY coletados em pimentão.
}

\author{
Monika Fecury Moura, Julio Massaharu Marubayashi; Tatiana Mituti; Ricardo Gioria; \\ Romulo Fujito Kobori; Marcelo Agenor Pavan; Renate Krause-Sakate.
}

UNESP-FCA (Botucatu), Rua José Barbosa de Barros, 1780, Departamento de Defesa Fitossanitária - Laboratório de Virologia Vegetal.

Autor para correspondência: Renate Krause-Sakate (renatekrause@fca.unesp.br)

Data de chegada: 30/05/2011. Aceito para publicação em: 13/02/2012.

1761

\section{RESUMO}

Moura, M.F; Marubayashi, J.M.; Mituti, T.; Gioria, R.; Kobori, R.; Pavan, M.A.; Krause-Sakate, R. Análise comparativa da região codificadora para a proteína capsidial de isolados de PepYMV e PVY coletados em pimentão. Summa Phytopathologica, v.38, n.1, p.93-96, 2012.

O Potato virus Y (PVY) e Pepper yellow mosaic virus (PepYMV) são as únicas espécies de potyvirus encontradas em pimenta e pimentão no Brasil. A região codificadora para a proteína capsidial de isolados de PepYMV e PVY coletados em pimentão, foi avaliada quanto à variabilidade e presença de motivos específicos aos potyvirus. A identidade da seqüência de aminoácidos na CP entre os isolados de PepYMV foi de $93 \%$ a $100 \%$, enquanto que para os de PVY $94 \%$ a
$98 \%$. Entre os vírus esta variou de $73 \%$ a $79 \%$. Foi observada variabilidade nas regiões conservadas da $\mathrm{CP}$. Todos os isolados de PepYMV seqüenciados não apresentaram o motivo DAG na $\mathrm{CP}$, relacionada a transmissão dos vírus por afídeos, enquanto que para as seqüências obtidas de PVY foi observada. Demais domínios como MVWCIENG, ENTERH, QMKAAA e PYMPRYG foram verificadas em ambas espécies.

Palavras-chave adicionais: pimentão, variabilidade, Potyvirus

\section{ABSTRACT}

Moura, M.F; Marubayashi, J.M.; Mituti, T.; Gioria, R.; Kobori, R.; Pavan, M.A.; Krause-Sakate, R. Comparative analysis of coding region for the coat protein of PepYMV and PVY isolates collected in sweetpepper. Summa Phytopathologica, v.38, n.1, p.93-96, 2012.

Potato virus $Y(\mathrm{PVY})$ and Pepper yellow mosaic virus (PepYMV) are the only potyvirus species found in pepper and sweetpepper in Brazil. The region encoding the coat protein of PVY and PepYMV isolates collected in sweetpepper was evaluated for the presence of specific motifs of potyviruses. The identity of the amino acid sequence in CP among PepYMV isolates was 93\% to $100 \%$, while for PVY was
$94 \%$ to $98 \%$. Among the viruses that ranged from $73 \%$ to $79 \%$. It was observed variability in the conserved regions of the CP. All PepYMV isolates sequenced did not show the DAG motif in the $\mathrm{CP}$, related to virus transmission by aphids, while for sequences of PVY this motif was observed. Other motifs, such MVWCIENG, ENTERH, QMKAAA and PYMPRYG were found in both species.

Keywords: sweet pepper, variability, Potyvirus

O pimentão (Capsicum annuum L.) é uma hortaliça cultivada em todo o Brasil, e encontra-se entre as dez olerícolas mais consumidas no país. A produção de pimentão é predominantemente em campo aberto, sendo que o Estado de São Paulo abrange cerca de $23,70 \%$ da área cultivada brasileira que é de 12.000 ha (11).

A suscetibilidade do pimentão a problemas fitossanitários causados por vírus ocasiona graves problemas à cultura. Entre os principais vírus do gênero Potyvirus pode-se citar Potato virus $Y$ (PVY), Pepper mottle virus (PepMoV), Tobacco etch virus (TEV), Pepper veinal mottle virus (PVMV) e Chilli veinal mottle virus (ChiVMV) (5,8) e Pepper yellow mosaic virus (PepYMV) (10), sendo que até o momento somente o PVY e o PepYMV são descritas no Brasil. O PVY no Brasil foi observado em pimentão pela primeira vez na década de 50 (6) e o PeYMV em 2002 (10). Ambos vírus são sorologicamente relacionados (7), e causam sintomas de mosaico indistinguíveis, tornando-os de difícil identificação visual.

Um dos critérios taxonômicos para classificação de espécies no gênero Potyvirus é comparação da identidade de sequência de aminoácidos codificadora para proteína capsidial (CP), que sendo inferior a $80 \%$, classifica isolados em diferentes espécies (3).

Neste trabalho foi analisada a CP de uma coleção de isolados de potyvirus provenientes das seguintes áreas produtoras de pimentão no Estado de São Paulo: Iacanga, Piraju, Lins e MogiMirim. O RNA total foi extraído a partir de plantas de fumo (Nicotiana tabacum cv. TNN), mantidas em casa de vegetação, utilizando-se o método de Bertheau (4) ou pelo kit de extração Total RNA Purification Norgen, seguindo as recomendações do fabricante. Para amplificação da CP tanto de PVY como PepYMV foi desenhado um par de oligonucleotídeos iniciadores denominados PepNib (5' GWTSGYYGMMTTGGATGATG 3') e PepUTR (5' AGTAGTACAGGAAAAGCC 3') que amplifica a região codificadora para a proteína capsidial (960 nts). A reação de RT-PCR para PVY e PepYMV foi realizada em uma só etapa e foram utilizados os Kits de PCR Master Mix das marcas PROMEGA, AMPLIQON e FERMENTAS. 
Para um volume final de $25 \mu \mathrm{L}$ foram adicionados: $5 \mu \mathrm{L}$ de produto de extração de RNA, 12,5 $\mu \mathrm{L}$ de tampão Master Mix, $1 \mathrm{mM}$ de cada oligonucleotídeo iniciador, 1 unidade da transcriptase reversa AMV (Avian myeloblastosis virus, Promega, $15 \mathrm{u} / \mu \mathrm{l})$ e água DEPC para completar o volume. O ciclo utilizado no termociclador (Mastercycler Gradient Eppendorf) consistiu em 30 minutos a $42{ }^{\circ} \mathrm{C}$, seguido de 2 minutos à $95{ }^{\circ} \mathrm{C}$ e 39 ciclos de $92{ }^{\circ} \mathrm{C} / 60$ segundos, $53{ }^{\circ} \mathrm{C} / 1$ minuto e $72{ }^{\circ} \mathrm{C} / 90$ segundos e finalizando com $72{ }^{\circ} \mathrm{C} / 10$ minutos. Foram retirados $5 \mu \mathrm{L}$ da reação de RT-PCR para análise em gel de agarose a $1 \%$ em tampão TBE $(0,1 \mathrm{M}$ de ácido bórico, 0,02 mM EDTA pH 8,3). Utilizou-se o marcador $1 \mathrm{~kb}$ Ladder da Invitrogen.

A região codificadora para a proteína capsidial de 8 amostras foi clonada no vetor comercial pGEM-T Easy (Promega). Inicialmente, os fragmentos obtidos por RT-PCR foram purificados através dos Kits comerciais Wizard SV Gel and PCR Clean-Up System (Promega) ou QIAquick PCR Purification Kit (Qiagen). A reação de ligação foi realizada adicionando-se $4 \mu \mathrm{L}$ de fragmento, $50 \mathrm{ng} / \mu \mathrm{L}$ de vetor, T4 DNA ligase $(10 \mathrm{mM}$ Tris- $\mathrm{HCl}, 50 \mathrm{mM} \mathrm{KCl}, 1 \mathrm{mM}$ DTT, $0,1 \mathrm{mM}$ EDTA, $50 \%$ glycerol), $5 \mu \mathrm{L}$ do tampão de ligação (60mM Tris$\mathrm{HCl}, 20 \mathrm{nM} \mathrm{MgCl} 2,20 \mathrm{DTT}, 2 \mathrm{mM}$ ATP, $10 \%$ PEG). A reação foi deixada durante a noite a $4{ }^{\circ} \mathrm{C}$. O vetor foi eletroporado em células competentes de Escherichia coli da estirpe XL1. Para verificar a presença do fragmento nos plasmídeos foi realizada uma clivagem com a enzima de restrição EcoR 1 (Invitrogen) por $1 \mathrm{~h}$ a $37^{\circ} \mathrm{C}$. As amostras com fragmento de tamanho desejado foram selecionadas para extração do plasmídeo pelo QIAprep Spin Miniprep Kit (QIAGEN), de acordo com as especificações do fabricante.

Para alguns isolados não foi necessário clonar o fragmento para posterior sequenciamento. A análise das sequencias foi realizada pelo programa Mega 4. 1 (12) e CLUSTAL Interative W (13).

Foram obtidas dez sequencias completas para PepYMV e duas para PVY. Os dois isolados de PVY apresentaram 94\% de identidade de aminoácidos com a sequencia de PVY depositada no GenBank sob numero PVY-LYE84.2. A identidade entre os isolados de PVY e PepYMV variou de $73 \%$ a $79 \%$. Os isolados de PepYMV apresentaram variações de porcentagem de identidade de aminoácidos de $93 \%$ a $100 \%$ entre si (Quadro 1).

Foram também analisados outros domínios específicos aos potyvirus (14). O domínio E(N/D)TERH (seqüência de nucleotídeos GARRAYACDGARMGNCAYRC) encontrada na CP dos potyvírus é considerado o mais conservado seguido pelos domímios QMKAAA, YAFDFYE, MVWCI(E/D)NG, $\mathrm{V}(\mathrm{W} / \mathrm{T}) \mathrm{MMDG}(\mathrm{D} / \mathrm{E} / \mathrm{N}), \quad$ (P/R/A)YMPRYG e (A/ S)SYN(ED)VD. As seqüências de aminoácidos ENTERH, QMKAAA, MVWCIENG e PYMPRTG foram observadas na região codificadora para a proteína capsidial dos isolados analisados de PVY e PepYMV. O domínio YAFDFYE foi observado em todas as seqüências, exceto para o isolado 23423 q de PepYMV em que foi substituído pela sequencia HAFDFYE. Para todos os isolados de PepYMV observou-se a falta da sequencia de aminoácidos DAG (Asparagina - Asp, Alanina - Ala, glicina - Gly) na região N-terminal da CP, altamente conservada entre os potyvirus e relacionada com a transmissão dos potyvirus por afídeos $(1,2)$. A seqüência DAG foi detectada em ambos os isolados de PVY analisados (Figura 1). Para PepYMV, este motivo não é essencial para transmissão pelos afídeos uma vez que transmissão pelas espécies Aphis gossypii e Myzus persicae já foi observada para um isolado de PepYMV (9). (Figura 1).

Neste trabalho foram evidenciadas as principais diferenças moleculares entre as CPs do PepYMV e PVY. A maioria dos domínios foram encontrados em ambas espécies, porém a que se destaca é a ausência do motivo DAG em todas as sequencias de PepYMV até então seqüenciadas.

\section{Agradecimentos}

À FAPESP pelo suporte financeiro e CAPES pela concessão da bolsa de mestrado para a primeira autora.

Quadro 1. Porcentagem de identidade da seqüência de nucleotídeos (diagonal superior) e da seqüência de aminoácidos (diagonal inferior) para a região codificadora para aproteína capsidial entre os isolados de PepYMV (252-29n, 268-32a, 254-6q, 257-6t, 249-291, 237-23t, 240-29c, 389-2c, 234-23q e 348) ; PVY (225-23j e 331); PepYMV AF348610 e PVY-LYE 84.2.

\begin{tabular}{|c|c|c|c|c|c|c|c|c|c|c|c|c|c|c|}
\hline & 251-29n & PepYMV & 268-32a & 254-6q & $257-6 t$ & 249-291 & $237-23 t$ & $240-29 c$ & $389-2 c$ & $234-23 q$ & PVY & $225-23 j$ & 348 & 331 \\
\hline $251-29 n$ & - & 94 & 94 & 93 & 93 & 99 & 99 & 99 & 93 & 99 & 65 & 65 & 98 & 65 \\
\hline PepYMV & 98 & - & 91 & 97 & 97 & 93 & 94 & 94 & 98 & 93 & 64 & 65 & 94 & 65 \\
\hline 268-32 a & 94 & 93 & - & 91 & 91 & 93 & 94 & 94 & 91 & 94 & 64 & 64 & 95 & 65 \\
\hline $254-6 q$ & 98 & 98 & 93 & - & 100 & 93 & 93 & 93 & 97 & 93 & 65 & 65 & 94 & 66 \\
\hline $257-6 t$ & 98 & 98 & 93 & 100 & - & 93 & 93 & 93 & 97 & 93 & 65 & 65 & 94 & 66 \\
\hline 249-291 & 98 & 97 & 93 & 97 & 97 & - & 99 & 98 & 93 & 98 & 65 & 65 & 98 & 65 \\
\hline $237-23 t$ & 99 & 97 & 94 & 97 & 97 & 97 & - & 99 & 93 & 99 & 65 & 65 & 98 & 65 \\
\hline $240-29 c$ & 99 & 98 & 94 & 97 & 97 & 98 & 98 & - & 93 & 99 & 65 & 65 & 98 & 65 \\
\hline $389-2 c$ & 97 & 98 & 93 & 98 & 98 & 96 & 97 & 97 & - & 93 & 64 & 65 & 94 & 65 \\
\hline 234-23q & 98 & 96 & 93 & 96 & 96 & 96 & 97 & 97 & 96 & - & 65 & 65 & 98 & 65 \\
\hline PVY & 76 & 78 & 77 & 78 & 78 & 75 & 75 & 76 & 78 & 74 & - & 93 & 65 & 93 \\
\hline $225-23 j$ & 74 & 75 & 76 & 76 & 76 & 74 & 74 & 74 & 78 & 73 & 94 & - & 65 & 99 \\
\hline 348 & 100 & 98 & 94 & 98 & 98 & 98 & 99 & 99 & 97 & 98 & 76 & 74 & - & 65 \\
\hline 331 & 75 & 76 & 76 & 76 & 76 & 74 & 74 & 75 & 79 & 74 & 94 & 98 & 75 & - \\
\hline
\end{tabular}




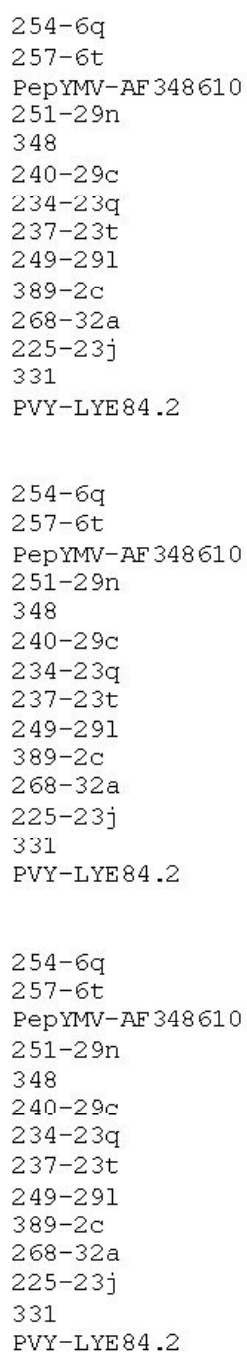

PVY-LYE 84.2

$254-6 q$

$257-6 t$

Pep YMV-AF 348610

251-29n

348

$240-29 \mathrm{C}$

$234-23 q$

$237-23 t$

$249-291$

$389-2 \mathrm{C}$

$268-32 a$

$225-23 j$

331

PVY-LYE 84.2
ADEKLAVLDAAE EDKKKRAKNEQPVDASNLRGKEKGVSTSKDNDVNTGTTGTF TVPRIKA 60 ADEKLAVLDAAE EDKKKRAKNEQPVDASNLRGKEKGVSTSKDNDVNTGTTGTE TVPRIKA 60 ADEKLAVLDAAE EDKKKRAKNEQPVDASNLKGKEKGV STSRDNDVNTGTTGTF TVPRIKA 60 ADEKLAVLDAAEEDKKKRAKNEQPADASNLKGKEKGVSTSRDNDVNTGTTGTFTVPRIKA 60 ADEKLAVLDAAE EDKKKRAKNEQPADASNLKGKEKGVSTSRDNDVNTGTTGTF TVPRIKA 60 ADEKLAVLDAAE EDKEKRAKNEQPADASNLKGKEKGVSTSRDNDVNTGTTGTETVPRIKA 60 ADEKPAVLDAAE EDKKKRAKNEQPADASNLKGKEKGV STSRDNDVNTGTTGTFTVPRIKA 60 ADEKLAVLDAAE EDKKKRAKNEQPADASNLKGEEKGVSTSRDNDVNTGTTGTE TVPRIKA 60 ADERLAVLDAAE EDKKKRAKNEQPADASNLQGKEKGV STSRDNDVNTGTTGTF TVPMDKA 60 ADDKLAVLDAAE EDKKKRAKNEQ PVDASNSKGKEKGV SASRDNDVNTGTTGTF TVPRIKA 60 ADEKLAVLDAAE EDKKKKARNEKPTEVPSLKGEEKEVSTARDNDVNTGTTGTETVPRIKA 60 ANDT---IDAGENSKK----DAKPAQGS IQQSPNK----AKEKDVNAGTSGTHTVPRIKA 49 GNDT---IDAGENSKK----DAKPAQGS IQQSPNK----AKEKDVNAGTSGTHTVPRIKA 49 ANDT---IDAGGSSKK----DAKPEQGS IQPTPNK----GKDKDVNAGTSGTHTVPRIKA 49

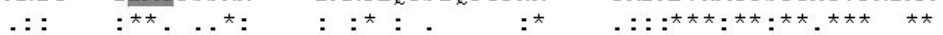

ITSKMRMPKVGGTTILNLDHLLTYTPQQIDI SNTR STHSQFDNNYAAVKNVYDVGDTEMQ 120 ITSKMRMPKV GGTT ILNLDHLLTYTPQQ IDISNTR STHS QFDNNYAAVKNVYDVGDTEMQ 120 ITSKMRMPKVGGTT ILNLDHLLTYTPQQ IDI SNTR STHSQFDNNYAAVKNVYDVGDAEMQ 120 ITGKMRMPKVGGTTILNLDHLLTYTPQQIDI SNTR STHSQFDNNYAAVKNVYDVGDTEMQ 120 ITGKMRMPKVGGTTILNLDHLLTYTPQQIDI SNTR STHSQFDNNYAAVKNVYDVGDTEMQ 120 ITGKMRMPKVGGTTILNLDHLLTYTPQQ IDISNTR STHSQFDNNYAAVKNVYDVGDTEMQ 120 ITGKMRMPKVGGTTILNLNHLLTYTPQQ IDISNTR STHSQFDNNYAAVKNVYDVGDTEMQ 120 ITGKMRMPKVGGTTILNLDHLLTYTPQQIDI SNTR STHSQFDNNYAAVKNVYDVGDTEMQ 120 ITGKMRMPKVGGTTILNLDHLLTYTPQQIDISNTR STHSQEDNNYAAVKNVYDVGDTEMQ 120 ITSKMRMPKVGGTT ILNLDHLLTYTPQQIDI SNTR STHSQFDNNYAAVKNVYDVGDTEMQ 120 ITGKMRMPKVGGTTILNLDHLLTYTPQQ IDISNTR STRNQFDNNYAAVKNVYDVGDTEMQ 120 ITSKMRMPKSKGAVALNLEHLLEYTPQQIDISNTRATQSQFDTNYDAVRVAYDIGENEMP 109 ITSKMRMPKSKGAAALNLE HLLEYTPQQIDI SNTRATQSQFDTNYDAVRVAYDIGENEMP 109 ITSKMRMPKS KGVAALNLEHLLEYTPQQIDISNTRATQSQFDTNYEAVRMAYDIGETEMP 109

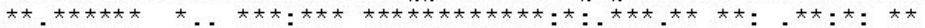

TIMNGLMNWC IENGTS PNINGVNTMMDGEEQVEYPLK PV IENSK PTF RQ IMAHF SDVAEA 180 TIMNGLMNWC IENGTS PNINGVNTMMDGEEQVEYPLK PV IENSKPTF RQ IMAHF SDVAEA 180 TIMNGLMNWCIENGTS PNINGVNTMMDGEEQVEYPLK PV IENSKPTE RQIMAHF SDVAEA 180 TIMNGLMNWC IENGTS PNINGVNTMMDGEEQVEYPLKPV IENSKPTE RQIMAHE SDVAEA 180 TIMNGLMVWC IENGTS PNINGVNTMMDGEEQVEYPLK PV IENSK PTE RQ IMAHE SDVAEA 180 TIMNGLMNWCIENGTS PNINGVNTMMDGEEQVEYPLK PV IENSK PTF RQ IMAHF SDVAEA 180 TIMNGLMVTCIENGTS PNINGVR TMTDGEEQVE YPLK PV IENSK PTF RO IMAHF SDVAEA 180 TIMNGLMNWC IENGTS PNINGVWTMMDGEEQVEYPLK PV IENSK PTF RQIMAHF SDVAEA 180 TIMNGLMNWCIENGTS PNINGVNTMMDGEEQVE YPLK PV IENSK PTF RQ IMAHF SDVAEA 180 TIMNGLMVWC IENGTS PNINGVNTMMDGEEOVE YPLK PV I EN SK PTF RO IMAHF SDVAEA 180 TIMNGLMNWC IENGTS PNINGVNTMMDGEEQVEYPLK PV IENSK PTLRQIMAHF SDVAEA 180 TVMNGLMVWC IENGTS PNV NGVWVMMDG SEQVEYPLK PIVENAKPTLRQIMAHE SDVAEA 169 TVMNGLMNACIENGTS PNVNGVWVMMDG SEQVEYPLKPIVENAK PTLRQ IMAHF SDVAEA 169 TVMNGLMVWC IENGTS PNINGVNVMMDGNEQVEYPLKPIVENAKPTLRQIMAHE SDVAEA 169

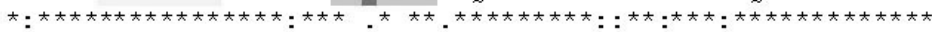

YIEMRNKKEPYMPRYGL IRNLRDLSLAR YAF DE YEVTSRT PTRAREAHIQMKAAALKSAQ 240 YIEMRNKKEPYMPR YGLIRNLRDLSLAR YAF DE YEVTSRTPTRAREAHIQMKAAALKSAQ 240
YIEMRNKKEPYMPRYGLIRNLRDLSLARYAF DF YEVTSRTPTRAREAHIQMKAAALKSAQ 240 YIEMRNKKEPYMPR YGL IRNLRDLSLAR YAF DE YEVTSRTPTRAREAHIQMKAAALKSAQ 240 YIEMRNKKEPYMPR YGLIRNLRDLSLAR YAF DE YEVTSRTPTRAREAHIQMKAAALKSAQ 240 YIEMRNKKEPYMPRYGLIRNLRDLSLARYAF DE YEVTSRTPTRAREAHIQMKAAALKSAQ 240 YIEMR NKKEPYMPR YGL IRNLRDLSLAR YAF DF YEVTSRTPTRAREAHIOMKAAALKSAQ 240 YIEMRNKKEPYMPR YGL IRNLRDLSLAR.HAF DF YEVTSRTPTPAREAHIQMKARALKSAQ 240 YIEMRNKKEPYMPR YGL IRNLRDLSLAR YAF DE YEVTSRTPTRAREAHIQMKAAALKSAQ 240 YIEMRNKKEPYMPR YGLIRNLRDLSLARYAF DF YEVTSRTPTRAREAHI OMKAAALKSAQ 240 YIEMR NKKEPYMPR YGL IRNLRDLSLAR YAF DF YEVTSRTPTRAREAHIOMKAAALKSAQ 240 YIEMR NKKEPYMPR YGLIRNLRDLSLAR YAF DF YEVTSRT PTRAREAHIQMKAAALKSAQ 240 YIEMR NKKE PYMPR YGL IR NLRD ISSAR YAF DF YEVTSRT PVRAREAHIOMKAAALKSAQ 229 YIEMRNKKEPYMPR YGLIRNLRDISLAR YAF DF YEVTSRT PVRAREAHIQMKAAALKSAQ 229 YIEMRNKKEPYMPRYGLIRNLRDISLARYAF DF YEVTSRTPVRAREAHIQMKAAALKSAQ 229

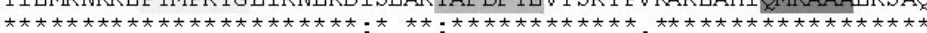

$254-6 q$
$257-6 t$
Pep $1 M v-A F 348610$
$251-29 n$
348
$240-29 c$
$234-23 q$
$237-23 t$

$\begin{array}{ll}\text { TRMFGLDGGISTQEENTERHTTEDVS PNMHTLLGVRNM } & 278 \\ \text { TRMFGLDGGISTQEENTERHTTEDVS PNMHTLLGVRNM } & 278 \\ \text { TRMFGLDGGISTQE ENTERHTTEDVS PNMHTLLGVRNM } & 278 \\ \text { TRMFGLDGGISTQEENTERHTTEDVS PNMHTLLGVKNM } 278 \\ \text { TRMFGLDGGISTQEENTERHTTEDVS PNMHTLLGVKNM } 278 \\ \text { TRMFGLDGGISTQEENTERHTTEDVS PNMHTLLGVKNM } 278 \\ \text { TRMFGLDGGISTQEENTERHTTEDVS PNMHTLLGVKNM } 278 \\ \text { TRMFGFDGGISTQEENTERHTTEDVS PNMHTLLGVKNM } 278\end{array}$

Figura 1. Alinhamento completo da região codificadora para a proteína capsidial entre os isolados coletados no campo comparados ao PepYMV (número de acesso AF348610) e PVY (número de acesso LYE84.2). 


\section{REFERÊNCIAS BIBLIOGRÁFICAS}

1. Atreya, P.B; Atreya, C.D; Pirone, T.P. Amino acid substitutions in the coat protein result in loss of insect transmissibility of a plant virus. Proceedings of the Nacional Academy of Sciences of the United States of America. n. 88, p-7887$7891,1991$.

2. Atreya, P.B; Lopez-Moya,J.J.; Chu, M.; Atreya, C.D.; Pirone, T.P. Mutational analysis of the coat protein $\mathrm{N}$-terminal amino acids involved in potyviral transmission by aphids. Journal of General Virology, London. n.76, p. 265-270, 1995.

3. Berger, P.H.; Adams, M.J.; Barnett, O.W.; Brunt, A.A.; Hammond, J.; Hill, J.H.; Jordan, R.L.; Kashiwazaki, S.; Rybicki, E.; S, N.; Stenger, D.C.; Ohki,S.T.; Uyeda,I.; van Zaayen, A. Valkonen, A.; Vetten, H.J. Potyviridae. In: Fauquet, C.M.; Mayo, M.A.; Maniloff, J.; Desselberger, U.; Ball, L.A. Virus Taxonomy: Eighth Report of the International Committee on Taxonomy of Viruses, p. 819-841, 2005.

4. Bertheau, Y.D., Frechon, I.K., Toth, L.J., Hyman. DNA amplification by polymerase chain reaction (PCR). In: PEROMBELON, M.C.M.; VAN DER WOLFF, J.M. Methods for the detection and quantification of Erwinia carotovora subsp. atroseptica on potatoes. Aberdeen: Scottish Crop Research Institute Occasional Publication, 1998.

5. Caranta, C., Lefebvre, V., Palloix, A. Polygenic resistance of pepper to potyviruses consists of a combination of isolate-specific and broad-spectrum quantitative trait loci. Molecular Plant-Microbe Interactions, Saint Paul, v.10, p.872-878. 1997.

6. Costa, A.S., Alves, S. Mosaico do pimentão. Bragantia, Campinas, v.10, p.95-96, Campinas, 1950.

7. Cunha, L.C.V. Da, Resende, R. De O., Nagata, T., Inoue-Nagata, A.K. Distinct features of Pepper yellow mosaic virus isolates from tomato and sweetpepper. Fitopatologia Brasileira, Brasilia, v.29, n.6, p.663-667, 2004.
8. Dogimont, C., Palloix, A., Daubze, A.M., Marchoux, G., Gebre-selassie, K. Pochard, E. Genetic analysis of broad spectrum resistance to potyvirus using doubled haploid lines of pepper (Capsicum annuum L.). Euphytica, Wageningen, v.88, p.231-239, 1996.

9. Gioria, R., Braga, R.S., Krause-Sakate, R., Roullier, C., Rosa, D.D., Moura, M.F., Souza-Dias, J.A.C, Sawazaki, H.E., Camargo, L.E.A., Rezende, J.A.M. Breakdown of resistance in sweet pepper against Pepper yellow mosaic virus in Brazil. Scientia Agricola, Piracicaba, v.66, n.2, p.267-269, 2009.

10. Inoue-Nagata, A. K., Fonseca, M.E.N., Resende, R.O.; Boiteux, L.S.; Monte, D.C.; Dusi, A.N.; Ávila, A.C. de; Vlugt, R.A.A. van der. Pepper yellow mosaic virus, a new potyvirus in sweetpepper, Capsicum annuum. Archives of Virology, Vienna, v.147, p.849-855, 2002.

11. Kobori, R.F.; Gioria, R.; Brunelli, K.R. Impacto potencial das mudanças climáticas sobre as doenças do pimentão no Brasil. In: GHINI, R.; HAMADA, E. Mudanças climáticas - Impactos sobre doenças de plantas no Brasil. Brasília - DF: Embrapa Informação Tecnológica, 2008, p.121-128.

12. Kumar, S., Tamura, K., Kakobson, I.B., Nei, M. Molecular evolutionary genetics analysis software. Bioinformatics, Oxford, v.17, p. 1244-1245, 2001.

13. Thompson, J.D.; Higgins, D.G.; Gibson, T.J. CLUSTAL W: improving the sensitivity of progressive multiple sequence alignment through sequence weighting, position-specific gap penalties and weight matrix choice. Nucleic Acids Research, Oxford, v.22, p. 4673-4680, 1994.

14. Zheng, L.; Wayper, P.J.; Gibbs, A.J.; Fourment, M.; Rodoni, B.C.; Gibbs, M.J. Accumulating variation at conserved sites in Potyvirus genomes is driven by species discovery and affects degenerate primer design. PloS ONE v.3, n.2, p. e1586. 2008. Disponível em: http://www.plosone.org. Acesso em: 16 de março de 2011. 